
U.S. DEPARTMENT OF COMMERCE

National Bureau of Standards

Center for Radiation Research

Washington, DC 20234

Daniel Gogny and Daniel Irakane

Centre d'Etudes de Bruyeres-le-Chatel 92542 Montrouge

CEDEX, France

July 1983 



\section{SIMPLE MODEL FOR THE QCD VACUUM}

Michael Danos,

Nastional Bureau of Standards, Washington, D.C. 20234, USA

and

Daniel Gogny and Daniel Irakane

Centre d'Etudes de Bruyeres-le-Chatel, 92542 Montrouge CEDEX, France

\section{Abstract}

By treating the high-momentum gluon and the quark sector as an in principle calculable effective Lagrangian we obtain a non-perturbative vacuum state for OCD as an. infrared gluon condensate. This vacuum is removed from the perturbative vacuum by an energy gap and supports a Meissner-0chsenfeld effect. It is unstable below a minimum size and it also suggests the existence of a universal hadronization time. This vacuum thus exhibits all the properties required for color confinement.

\section{Introduction}

By now it is widely believed that the confinement in QCD, in analogy with superconductivity, results from the existence of a physical vacuum which is removed from the remainder of the spectrum by an energy density gap and which exhibits a Meissner-Ochsenfeld effect. 1 More particularly, it is believed that these characteristics of the physical vacuum result from the infrared properties of QCD. With this in mind we will construct in this paper a simple model for QCD which concentrates on the low-energy part and parametrizes the high-energy part of the theory, and we will show that this simple model has a non-perturbative solution for the vacuum which indeed possesses the desired characteristics. Even though this model is extremely simple we believe that it is sufficiently accurate to reproduce the essential qualitative features of the QCD vacuum. Furthermore, the accuracy of the model can be improved; in principle the model could be enlarged in a straight-forward manner to yield approximations to the actual solution of the QCD vacuum problem, and hence, to QCD. 
The most extensive recent treatments of the confinment problem are based on the evaluation of Wilson loops. ${ }^{2,3}$ This method avoids the explicit introduction of the structure of the vacuum. The properties of the vacuum itself are discussed in several papers which show that it can have the character of a superconductor, i.e., that a superconductive vacuum is compatible with QCD. ${ }^{4}$ Our paper supplements these references in that it provides an explicit construction of the vacuum which demonstrates that a superconductive QCD vacuum not only is possible but actually exists.

The present paper is organized as follows. In section II we define the model and show how it follows from the QCD Lagrangian. We perform the analysis in the Hamiltonian formalism in the Schroidinger picture, discussed in detail in references 5-7. The drawback of not being manifestly covariant is outweighed by the fact that this treatment lends itself immediately to achieving non-perturbative solutions. In this section we show how the property of anti-screening inherent in QCD leads to a aap, and we discuss the conditions for the existence of the gap.

Even though an exhaustive description of the Meissner-Ochsenfeld effect requires a correct treatment of the vacuum-baq interface we show in section III that our present simple model already points toward the existence of that effect. In section IV we discuss the effect of our neglect of the center-of-mass motion and indicate that this leads to an error on the conservative side, i.e., that our treatment yields a lower limit on the gap size. We sketch the manner in which the physical vacuum condenses from the perturbative vacuum in section $V$, using as an example the decay of a $\pi^{0}$ into two photons. We find indications for the existence of a universal time constant for this process, or, equivalently, for a universal hadronization time, similar for all hadrons. Finally, we give a summary of our results. 
II. The Gap

In order for the results of the model theory to have any bearing on QCD, the model should contain as many as possible of the essential ingredients of QCD. We now discuss our reasoning.

Firstly, we share the belief that the gauge sector by itself,

$$
\begin{gathered}
L_{G}=-\frac{1}{4} F_{\mu \nu}^{a} F_{\mu \nu}^{a} \\
F_{\mu \nu}^{a}=\partial_{\mu} A_{\nu}{ }^{a}-\partial_{\nu} A_{\mu}{ }^{a}-g f^{a b c}\left(A_{\mu}{ }^{b} A_{\nu}{ }^{c}\right)
\end{gathered}
$$

is incapable of yielding a gap since the Lagrangian (1) contains no scale. Namely, if one should obtain from (1) a gap, say, $\Delta=300$, then this result would mean simultaneously $\Delta=300 \mathrm{eV}$ and $\Delta=300 \mathrm{GeV}$, which clearly is impossible. In fact, the Fermion sector of QCD does contain scale factors in the form of the quark mass terms. (The origin of the quark masses is still rather mysterious; however this need not concern us here.) In a covariant treatment the Fermion sector would intrude into the Boson sector via modifications of the Boson propagators and the vertex functions. We now shall describe how we approximate this effect in our treatment.

As explained in references 5-7, one may derive in the Schrödinger picture of the fields a field-theoretic Schrodinger equation from the fieldtheoric Lagrangian:

$$
H|S\rangle=E|S\rangle
$$

where $|S\rangle$ is the state vector describing an eigenstate of the system. We break the Hilbert space into two parts and rewrite eq (3) as 


$$
H\left(\begin{array}{l}
F \\
G
\end{array}\right)=\left(\begin{array}{ll}
X & Y \\
y^{+} & Z
\end{array}\right)\left(\begin{array}{l}
F \\
G
\end{array}\right)=E\left(\begin{array}{l}
F \\
G
\end{array}\right)
$$

from which one may eliminate $G$ to obtain

$$
X F+Y \frac{1}{E-Z} Y+F \equiv H_{0} F+H_{e f f} F=E F
$$

The form of the operators $X, Y, Z$ depends on the division of the Hilbert space, and in QCD also on the choice of the gauge. We shall take the space $F$ to consist of that part of the Hilbert space which contains only low momentum transverse gluons, while $G$ contains the rest of the Hilbert space, i.e., quarks, longitudinal gluons and high momentum transverse gluons. The precise specification of $F$ will begiven below.

Supposedly the confinement problem is an infrared, i.e., low momentum phenomenon. Hence it should be possible to divide the the Hilbert space such that all high-momentum phenomena are taken care of in the space $G$. In other words, one may hope that an approximate separation, as ymptotic freedominfrared slavery, is possible in the sense that the "renormalized" parameters calculated solely in the part $G$ of the Hilbert space are reasonably independent of the size of the part $F$, for "sufficiently small" $F$, while at the same time $F$ is "sufficiently large" as far as the description of the infrared properties is concerned. As a consequence of this separation only the part $F$ of the Hilbert space must be treated non-perturbatively; the part G could be treated by perturbation methods. These considerations form the basis of our model.

Before discussing the specifics of our model we specify our choice of the representation of the transversal vector field operators. ${ }^{5-7}$ To wit, we expand the (Schrodinger picture) field operators in terms of the complete, 
orthonormal, discretized set of vector spherical functions of good angular momentum, $\mathrm{J}$, and parity, specified by the index $\mathrm{k}=$ magnetic or electric, i.e., the analogue of the multipole expansion of electrodynamics, augmented by the color index a:

$$
A=\Sigma\left(a_{J k u m a} K_{J k u m a}(r)+h_{.} c_{0}\right)
$$

The creation-annihilation operators fulfill

$$
\left[a_{J K u m a}, a_{J^{\prime} K^{\prime} \nu^{\prime} m^{\prime} a^{\prime}}+\right]_{-}=\delta_{J J^{\prime}} \delta_{K K^{\prime}} \delta_{\nu \nu^{\prime}} \delta_{m m^{\prime}} \delta_{a a^{\prime}}
$$

The discretization has been achieved by forming wave packets in the momentum $|k|$ with a set of orthonormal weight functions specified by the index $v$. This way the functions $k_{\nu}$ have a well defined finite mean square momentum $\left\langle k^{2}\right\rangle$, which depends on $v$, and limited spread $\left\langle k^{4}\right\rangle-\left\langle k^{2}\right\rangle^{2}$. At the same time the functions $k_{\nu}$ have a limited size in position space, i.e., both $\left\langle r^{2}\right\rangle$ and $\left\langle r^{4}\right\rangle-$ $\left\langle r^{2}\right\rangle^{2}$ have well-defined, finite values. We describe this size by the parameter $\ell^{2}=\left\langle r^{2}\right\rangle$ computed for $v=1$.

At this point we must specify the model. We shall test whether employing a non-perturbative solution suffices for developing an energy gap for the vacuum. To that end we make the simplest possible choice for $F$, and we consider the particular coherent pairing state constructed with one of the components of eq (6) only,

$$
F^{\prime}|0\rangle=e^{-\theta / 2\left(a_{J K \nu}^{+} \cdot a_{J_{K \nu}}^{+}\right)}|0\rangle
$$


where the dot indicates formation of a singlet in both Minkowski and color spaces and, of course, we take $\nu=1$. We have omitted in eq (8) the time dependence; it will have to be added to achieve the complete Schroidinger picture state vector.

This state contains an unspecified number of pairs. It connot be achieved in a perturbative treatment as it is connected with the perturbative vacuum $|0\rangle$ only by an infinite number of applications of the Hamiltonian. The parameter $\theta$ is a variational parameter which will be used to find a (local) minimum of the Hamiltonian. For technical reasons, similar to those in superconductivity it is advantageous to replace eq (8) by

$$
\begin{aligned}
& F|0\rangle=e^{-\theta / 2 B_{J K \nu}}|0\rangle \equiv|V\rangle, \\
& B_{J_{K \nu}}=\left(a_{J_{K \nu}}^{+} \cdot a_{J_{K \nu}}^{+}-a_{J_{K \nu}} \cdot a_{J_{K V}}\right) .
\end{aligned}
$$

This operator, (10) being anti-Hermitian, respects the normalization of $|0\rangle$. At this point we should discuss the meaning of the Boson pairing state eqs (8) or (9). A general creation term of the series (9) has the form $\left(a^{+} \cdot a^{+}\right)^{n}$, i.e., it is a symmetric product of pairs, where each pair has angular momentum and color zero (it is a singlet state). The solution thus would describe a finite volume $\ell^{2}$ filled with the "physical" vacuum, floating in a sea of the "bag" vacuum, i.e., a situation which may arise during the cooling off of a quark-gluon plasma. Being constructed from a discretized set of states the total momentum of an n-pair component, i.e., $k=k_{1}+k_{2}+\ldots$ $k_{2 n}$ does not vanish. This "spurious center of mass motion" in principle can be taken care of as explained e.g., in references 5 and 7; as we will discuss below, this is, however, of no importance in the context of our question, viz., the existence of a gap. 
We now describe the form of the effective Hamiltonian, eq (5), rewritten as the variational problem

$$
\begin{gathered}
\langle F|X| F\rangle+\left\langle F\left|Y \frac{1}{E-Z} Y+\right| F\right\rangle=E \\
\delta\left\{\langle F|X| F\rangle+\left\langle F\left|Y \frac{1}{E-Z} Y+\right| F\right\rangle\right\}=0 .
\end{gathered}
$$

The first term of (11) is simply

$$
H_{0}=\langle|k|\rangle a^{+} a \equiv \omega a^{+} a,
$$

which defines the energy $\omega$. It is the second term where the essential model assumptions have to be made. To begin with, the form of $F$ requires that the number of Fock space operators be even. The simplest possibility is to allow four operators, as shown in figure 1a. In principle, the effect of very complicated high-order graphs can be contained within this limitation.

To write the simplest possible four-field vertex we take a hint from the effective Euler-Heisenberg Lagrangian for the vacuum polarization in QED, 8, 9 which reads

$$
\int d^{3} x H_{e f f} Q E D=\frac{2}{15 \pi^{2} m^{4}} \int d^{3} x E^{4}
$$

This form was derived as a local approximation to the non-local higher order corrections, and hence is valid for low momentum transfers, $\underline{i} . \underline{e}$. , in the long wavelength limit. Here $\mathrm{m}$ is the electron mass. Equation (14) is written in terms of the field strengths $E$. We now assume for our case, replacing the field strengths by the potentials, the effective force to be of the form 


$$
Y \frac{1}{E-Z} Y^{+} \rightarrow H_{I}=g^{4} \varepsilon(\ell M)-4 \int d^{3} x:[A \cdot A][A \cdot A]: \equiv \alpha \int d^{3} x:[A \cdot A]^{2}: .
$$

In this expression $M$ is the scale reflecting the quark masses, which, in principle, could be computed; $\ell$ is the above defined size parameter of the functions $K$; $\varepsilon$ is a sign to indicate "attractive" or "repulsive," which, as we will show below, leads to infrared slavery or infrared freedom, and $\mathrm{g}$ is the effective couping constant, appropriate to the momenta relevant for the space $F$. It again contains a scale, $\Lambda$, which is associated with the quark masses, and can be written, as usual,

$$
q^{2}=\frac{\alpha_{0}}{\log \frac{k^{2}}{\Lambda^{2}}},
$$

where $k^{2}$ is some typical momentum transfer. The form (14) is supposed to encompass also the effect of the 4-field term of order $g^{2}$ contained in eqs (1), (2). This completes the description of the model.

We note here that in QED the sign $\varepsilon$ is positive, consistent with shortdistance screening of the charges. In QCD the vacuum polarization has the anti-screening $\operatorname{sign} \varepsilon=-1$; we can expect that the term (14) representing a more general class of graphs will retain this sign.

Note that for $\varepsilon=-1$ our effective Hamiltonian is not positive definite, i.e., the Hamiltonian $\mathrm{H}_{0}+\mathrm{H}_{\mathrm{I}}$ is not bounded from below. Therefore, one cannot expect to achieve a stable vacuum state. This, of course, is also true if one takes for $H_{I}$ the attractive QCD Coulomb force. * Since the original

\footnotetext{
*This is in contradiction with the result of reference 10 where a stable minimum for the vacuum state was reported.
} 
Hamiltonian presumably is bounded from below the effective Hamiltonian (13), (14) most certainly is an insufficient representation of the effective force $H_{\text {eff }}$ of (5). To achieve a bounded Hamiltonian one has to continue the expansion implied by (14). To that end we add the sixth-order term

$$
H_{I I}=g^{6} M V \int d^{3} x:(A \bullet A)(A \bullet A)(A \bullet A): \equiv B \ell^{2} \int d^{3} x:(A \bullet A)^{3}:
$$

where we have introduced the dimensionless interaction strength parameter $B$. As we will show below the nature of the results is independent of the actual magnitude of $B$ over a very wide range of values.

In order to solve our model we introduce the Boson analogue of the Bogoliubov quasi-particle transformation 11,12 by writing

$$
\begin{aligned}
& b=c a+s a^{+} \\
& b^{+}=s a+c a^{+}
\end{aligned}
$$

together with the demand

$$
b|V\rangle=0
$$

where $|V\rangle$ is taken to be of the form (9). A straight-forward calculation yields the conditions

$$
\begin{aligned}
& c=\cosh \theta \\
& s=\sinh \theta
\end{aligned}
$$


for eq (19) to be fulfilled. We now look for the best solution achievable with the form (9) by searching for the minimum energy:

$$
\frac{\partial E}{\partial \theta}=\frac{\partial}{\partial \theta}\langle V|H| V\rangle=0
$$

To evaluate (21) we re-write the Hamiltonian in terms of the operators $b, b^{+}$,

$$
\frac{H}{S \pi \omega}=\left[\frac{1}{x}+\alpha P+B Q(x-1)\right](x-1)^{2}+R \text {. }
$$

Here $S$ is the statistical factor

$$
S=2 \times\left(N^{2}-1\right)=2 \times 8=16
$$

where the indicated numerical value is that valid for SU(3). Further,

$$
x=e^{2 \theta}
$$

and

$$
\begin{gathered}
P=3\left(S+\frac{2}{3}\right) \frac{1}{(\ell \omega)^{2}}=\frac{50}{(\ell \omega)^{2}} \\
Q=\frac{3}{2}\left(S+\frac{2}{3}\right)\left(S+\frac{4}{3}\right) \frac{1}{(\ell \omega)^{3}}=\frac{26}{3} \frac{1}{\ell \omega} P \\
R=\frac{4}{S} \frac{1}{x}: b^{+} b:+\frac{3}{S} \frac{1}{(\ell \omega)^{3}}\left[\alpha+3 \beta\left(S+\frac{2}{3}\right) \frac{1}{\ell \omega}(x-1)\right] x^{2}:[D \cdot D]^{2}: \\
+\frac{4 B}{S} \frac{1}{(\ell \omega)^{4}} \times:[D \cdot D]^{3}:
\end{gathered}
$$

with

$$
D=b+b^{+}
$$


Owing to eq (19) the contribution of $R$ to the expectation value (21) vanishes. Since $x>0$ one sees from (22) that indeed a non-trivial solution, i.e., $\theta \neq 0$ requires $\alpha<0$. Also, in that case for $\beta=0$, as expected, $H \rightarrow-\infty$ for $x \rightarrow \infty$. On the other hand, any $\beta>0$ stabilizes the Hamiltonian. In figure 2 we show the function defined by (22) with $R=0$ as a function of $\theta$ for several values of the parameters $\alpha$ and $\beta$. Note that the principal minimum at $\theta<0$ is essentially independent of the value of $\beta$ as long as $\beta$ is larger than the value for which the secondary minimum is higher than the principal minimum, i.e., if

$$
B Q>1 \text {. }
$$

Also note, that the principal minimum, i.e., the minimum at $x<1$, i.e., $\theta<0$, occurs for

$$
\alpha p<-1
$$

Recalling (26) one sees that the inequality (29) very probably is fulfilled if (30) is fulfilled. Of course, a definite statement must await the required QCD calculations.

At this point a discussion of the physical implications is in order. Regarding (30) and (15) superficially, it seems that the size of the physical vacuum is limited, i.e., that by increasing $\ell,|\alpha|$ diminishes and at some point (30) can not be fulfilled. This conclusion is, however, erroneous. Namely, when enlarging $\ell$, the limitation of the expansion of the fields to the single term, (9), (10), becomes untenable, and an increasingly larger number of terms of the expansion (6) must be used, and will contribute to the 
physical vacuum state. This in effect will be equivalent to increasing the value of the statistical factor $S$, eq (23), and will compensate for the dependence of the individual matrix elements on $\ell$. On the other hand, when decreasing $\ell$ the effective coupling constant, eq (16), will decrease, finally leading to the violation of (30). Again, unfortunately we must defer a quantitative discussion to the time that sufficietly accurate QCD calculations become available. Finally we note that $|V\rangle$ is not an eigenstate of $H$ since the Hamiltonian connects states which differ in the number of quasi-particle pairs, i.e.,

$$
\left\langle V_{n}|H| V_{n \pm v}\right\rangle \neq 0, \quad v=1,2,3
$$

where

$$
\left|V_{n}\right\rangle=\left(b^{+} b^{+}\right)^{n}|V\rangle
$$

By diagonalozing $H$ in the space of the functions $\left|V_{n}\right\rangle$ one can obtain an improved form for the physical vacuum. In view of the form (9) of $|V\rangle$ it might be advantageous in the diagonalization of $H$ to use instead of (32) the form

$$
\left|\tilde{V}_{n}\right\rangle=L_{n}^{(1 / 2)}(\theta B) e^{-(\theta / 2) B}|0\rangle
$$

which may be a better approximation to an n-quasi-pair state than (32). In eq $(33) L_{n}^{(1 / 2)}(x)$ is the (normalized) Laguerre polylnomial and $B$ is given by eq (10), dropping all indices. We shall not perform this calculation here in view of the absence of a QCD calculation for the interaction strengths of the effective Hamiltonian (22). 
III. The Meissner-Ochsenfeld Effect

In order to achieve color confinement the physical vacuum $|V\rangle$ must support a color Meissner-Ochsenfeld effect, i.e., it must follow from the properties of $|V\rangle$ that in the bulk a presence of a color field is incompatible with the existence of $|V\rangle$. Of course, in analogy with superconductivity one must expect that color fields penetrate a certain distance into the physical vacuum in order to achieve the shielding of the color electric and magnetic charges and currents. In other words, the understanding of the confinement problem demands an understanding of the transition region $|0\rangle \leftrightarrow|V\rangle$, i.e., of the structure of the "bag" surface, or, equivalently, of the vacuum-bag interface.

In order to be capable of describing both an interior and a surface one must retreat from the extreme simplification (10) for $F$. Therefore, in order to be able to describe the Meissner-Ochenfeld effect, the single-particle function in (6) must be made more flexible, at. least by replacing $k_{v}$ by

$$
K_{v} \rightarrow G=\sum_{n=1}^{N} c_{v} K_{v}
$$

The $c_{v}$ are variational parameters to be determined by a self-consistent solution of the now shielded external field and $G$, as explained, for example, in Chapter II.4 of Reference [12]. The size parameter $\ell$ in the functions $K$ here will have to be chosen to be of the order of the radius $R$ of the vacuum droplet, while the required number of harmonics, $N$, will be given by the ratio $R / d$, where $d$ is the surface thickness. By changing $N$ and finding where the results stabilize one thus can determine the surface sturcture. 
After having obtained this new set of "one-body" fields, eq (34), one can repeat the analysis of the previous section to obtain the Bogoliubov quasiparticle vacuum in terms of the function (9). This vacuum again supports quasi-particles, and shows a gap in the energy spectrum. As is well known, these characteristics are sufficient to insure the existence of the MeissnerOchsenfeld effect. 12 The proof of that reference that in first order in the external field no quasi-particles are excited thus holds also in the present case and this completes the demonstration that our model vacuum has the expected characteristics.

One could carry the analysis further to compute the critical field strength (see, e.g., Ref. 12, Chapter II.5), but we shall not do that here in view of the absence of QCD calculations of the coefficients $\alpha$, $\beta$ of eq (15), (17)

IV. Center-of-Mass Motion and Boost of the Solutions

Upon solving eqs (21) with (9), (10) one achieves an approximation to the solution of eqs (11) and (12). Being only an approximation the solution inherently will have inaccuracies. We now will discuss the properties of these solutions.

The first point concerns the energy of the solution $|V(t)\rangle$, which here we take to be (9) augmented by its time dependence. The expectation value (22),

$$
\langle V(t)|H| V(t)\rangle=E_{V}<0
$$

cannot actually be the physical eigenvalue of the vacuum. Even more strongly, in the utilized quantized form all energies must be non-negative. The result (35) simply implies the need for a kind of gauge transformation. It is 
equivalent to the case of classical electrodynamics where one can shift the energy scale arbitrarily up or down by the addition of a constant scalar, i.e., time-like, potential, i.e., by a global gauge transformation. The same $c$ an be done here by a re-definition of the phase of the state vector $|V\rangle$ :

$$
\left|V_{0}\right\rangle=e^{+i E_{v} t}|V(t)\rangle
$$

with this phase the new state vector obeys

$$
\frac{\partial}{\partial t}\left|V_{0}\right\rangle=0
$$

Remembering that we work in the Schrodinger picture this then yields for the vacuum energy

$$
E_{0}=0
$$

At the same time the perturbative vacuum acquires the energy $\left|E_{v}\right|$. More precisely, the number $\left|E_{v}\right|$ actually is the energy needed to replace $\left|V_{0}\right\rangle$ by $|0\rangle$, and since in our model system the state $\left|V_{0}\right\rangle$ occupies only a volume $\ell^{3}$ in position space, the number $\left|E_{v}\right|$ actually represents an energy density

$$
\rho=\frac{\left|E_{v}\right|}{\ell^{3}}
$$

With this normalization one might think that the state $\left|V_{0}\right\rangle$ would be boost-invariant, if each pair in (10) would have $\vec{k}_{1}+\vec{k}_{2}=0$. Namely, the moment um four-vector would be 


$$
P=(E, \vec{k})=(0,0)
$$

which indeed remains the same in all frames. However, the question of the CM motion is somewhat more subtle.

Namely, it is relatively easy to achieve a state where the CM momentum vanishes. For example, the gluon pair $\vec{e}_{x} e^{i k z_{1}} \vec{e}_{-x} e^{-i k z_{2}}$, i.e., a two-gluon plane wave state where the gluons have opposite polarizations and momenta, indeed has $k_{1}+k_{2}=0$ and vanishing field strength. Of course, its center of mass position is not fixed. The difficulty arises from the fact that in a finite-size system one requires the knowledge of the $C M$ position, since the $C M$ position must coincide in all components of the state $\left|V_{0}\right\rangle$. For a state containing $\mathrm{N}$ particles the $\mathrm{CM}$ position operator is an $\mathrm{N}$-body operator. ${ }^{5,7}$ Since our state $\left|V_{0}\right\rangle$ is a superposition of configurations containing arbitrary numbers of particles the calculation of the CM.position would be arbitrarily complicated. However, as our present model has only a qualitative significance the only question of interest is, whether or not the inaccuracy associated with the CM problem would invalidate our qualitative result, $\underline{i} . \underline{e}$. , the existence of a gap. We now would like to present an argument which indicates that this in fact does not happen, i.e.., that only a numerical inaccuracy results from the errors in the treatment of the CM motion.

Our reasoning is as follows. The Hamiltonian, eqs (13) and (14), is translationally invariant. Any inaccuracies of the results associated with the $C M$ motion arise from the truncation of the Hilbert space. Now, if two components of the state $\left|V_{0}\right\rangle$ have $C M-s$ which do not coincide then one must expect that the size of the matrix element of $H$ is diminished from its correct value, i.e., the value which would obtain when the CM-s coincide. Hence the 
computed off-diagonal energy is a lower limit to the actual off-diagonal energy, $\underline{i}$.e.., the error is on the "conservative" side, and the calculated gap represents a lower limit on the actual gap size of the model.

\section{Time development}

An important characteristic of the physical vacuum is the time it takes for it to be formed. For example, upon the decay of a hadron into leptons or photons the perturbative vacuum disappears by being replaced by the physical vacuum. Since this process involves a change in the structure it can not happen instantaneously. We shall here sketch the description of this process, and, in particular, we consider the development of our physical vacuum droplet, say, upon the annihilation of a qqं pair, e.g., a $\pi^{0}$, into photons.

The reason one has to consider this process is that the vacuum state |V> is connected with the perturbative vacuum $|0\rangle$ in a non-perturbative manner. The usual procedure of the graph expansion of the time-dependent perturbation theory is thus not feasible. A direct treatment is therefore called for.

Quite generally, maintaining the restriction (9) to a single one-particle state, the vacuum state vector is given by the expansion into quasi-pair states (33)

$$
|V(t)\rangle=\sum_{n} C_{n}(t)\left|\tilde{V}_{n}\right\rangle
$$

where the parameter $\theta$ contained in (9), (10), may be taken to be either timedependent or time-independent owing to the completeness of the set (33) with fixed $\theta$. At any rate, the time-dependence is governed by

$$
\frac{\partial}{\partial t}|V(t)\rangle=-i H|V(t)\rangle
$$


This Schrödinger equation can be solved by a time-dependent Bogoliubov transformation, i.e., by the transformation (18) where the coefficients $c$, s, are taken to be $c$-number functions of $t$. The condition (19) then can be fulfilled at each instant in time, and all results remain formally the same; onlv the parameters become functions of time, as already indicated in (41). In particular, the form of the Hamiltonian (22) remains the same. Note that the time-dependence of the unitary transformation (18) renders the Hamiltonian only superficially time-dependent. Hence all the conservation laws remain valid; in particular $\frac{\partial H}{\partial t}=0$, and energy is, of course, conserved.

We now sketch the solution of the time-dependence of the vacuum by invoking the sudden approximation. To that end we consider $\theta$ in (41) to have the asymptotic value given by (21), and we have at $t=0$, i.e., at the time of the emission of the annihilation photons, the perturbative vacuum,

$$
|V(0)\rangle=|0\rangle \approx \sum C_{n}(0)\left|\tilde{V}_{n}\right\rangle
$$

Since the individual components of (43) have a time-dependence given by their respective energies the higher terms will interfere away in a time given by the quasi-pair excitation spectrum. An exponential decay into the physical vacuum arises here in view of the continuum of the final state (of the emitted $\gamma$-rays in our example) in the familiar manner upon integration over the energy.

Note that this time development is associated only with the characteristics of the vacuum state. Hence this time constant should be essentially the same for all processes, or, in the time-reversed sense, all hadronization times should be about the same. The only differences can arise from slight differences in the initial conditions (43), which a priori can not be excluded. 
In summary, we have demonstrated that in our model which we believe reflects with sufficient accuracy the infrared aspects of QCD, a non-perturbative vacuum exists which exhibits an energy gap and which repells color electric and magnetic fields. Thus this vacuum has all the characteristics required for color confinement. 


\section{REFERENCES}

1. The suggestion that a superconductivity-type vacuum may be the origin of conf inement has been made already a very long time ago: Y. Nambu and G. Jona-Lasino, Phys. Rev. 122, 345 (1961); see also J. Schwinger, Phys. Rev. 125, 397 (1962), 128, 2425 (1962). These papers, of course, were not formulated in the modern language of QCD.

2. K. G. Wilson, Phys. Rev. D10, 2445 (1974): For a recent summary of lattice gauge theory resuTts see, e.g., C. Rebbi in Proc. 21, International Conference on High Energy Physics, Paris (1982) (to be published).

3. P. Hasenfratz and I. Montvay, Phys. Rev. Lett. 50, 309 (1983).

4. R. Fukuda, Prog. Theor. Phys. 67, 648 (1982), and references therein.

5. M. Danos and V. Gillet, Relativistic Many-Body Bound Systems, NBS Monograph 147, Washington, D.C. (1975).

6. M. Danos and V. Gillet, in Mesons in Nuclei, M. Rho and D. Wilkinson, eds., p. 839, North Holland Publishing Co., Amsterdam, New York, Oxford (1979).

7. M. Danos, V. Gillet, and M. Cauvin, Methods in Relativistic Nuclear Physics, North Holland Publishing Co., Amsterdam, New York, Oxford (1983).

8. H. Euler and W. Heisenberg, Z. f. Phys. 98, 714 (1936).

9. M. Danos and D. Zanon, Lett. Nuovo Cim. 31, 61 (1981).

10. R. Fukuda and T. Kugo, Progr. Theor. Phys. 60, 565 (1978).

11. See, for example, P. Ring and P. Schuck, Appendix E in The Nuclear ManyBody Problem, Springer Verlag, New York, Heidelberg, Berlin (1980).

12. J. M. Blatt, Theory of Superconductivity, Academic Press, New York, London (1964). 


\section{FIGURE CAPTIONS}

Fig. I (a) Graphs represented by (14).

(b) Graphs not contained in (14).

Fig. 2 Vacuum energy, eq (22) with $R=0$, for some values of the parameters. $\alpha /(\ell \omega)^{2}=-0.1 . \quad B /(\ell \omega)^{3}$ : (a) 0.006 ; (b) 0.1 ; (c) 10 . 


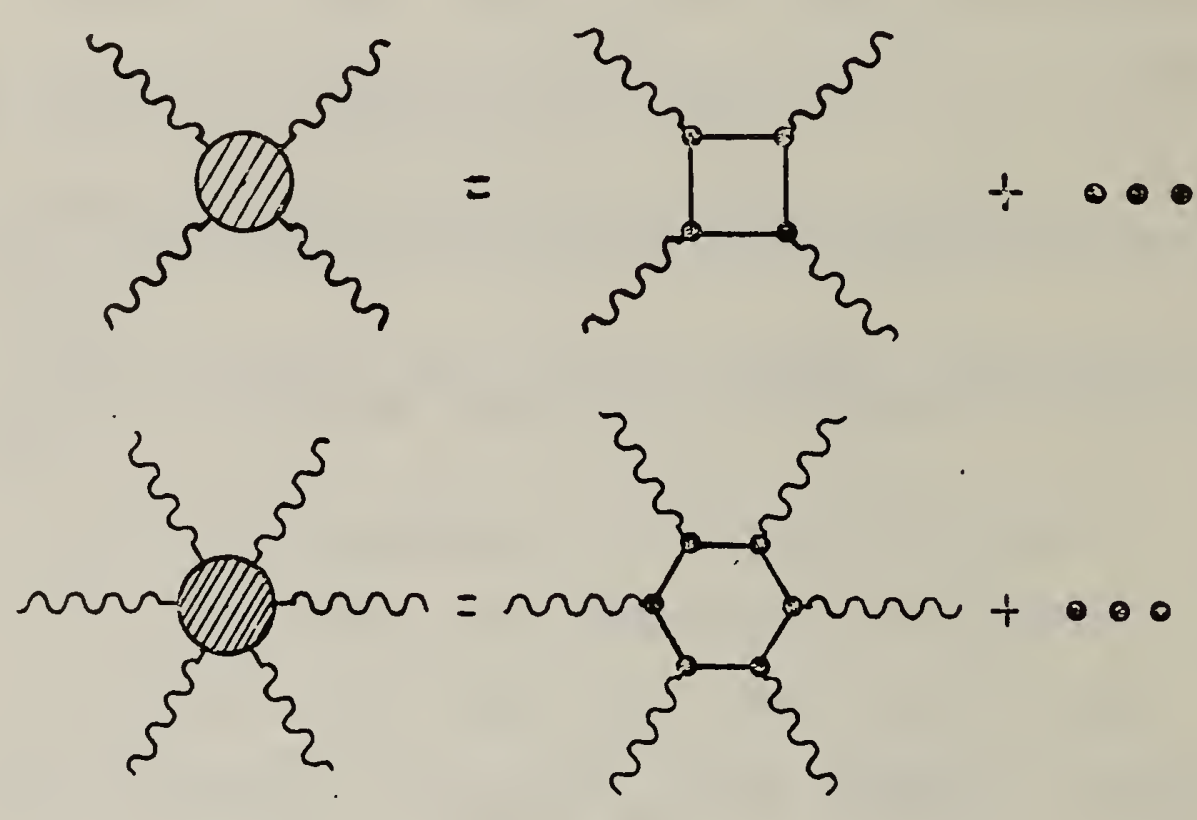

(a)

(b) 


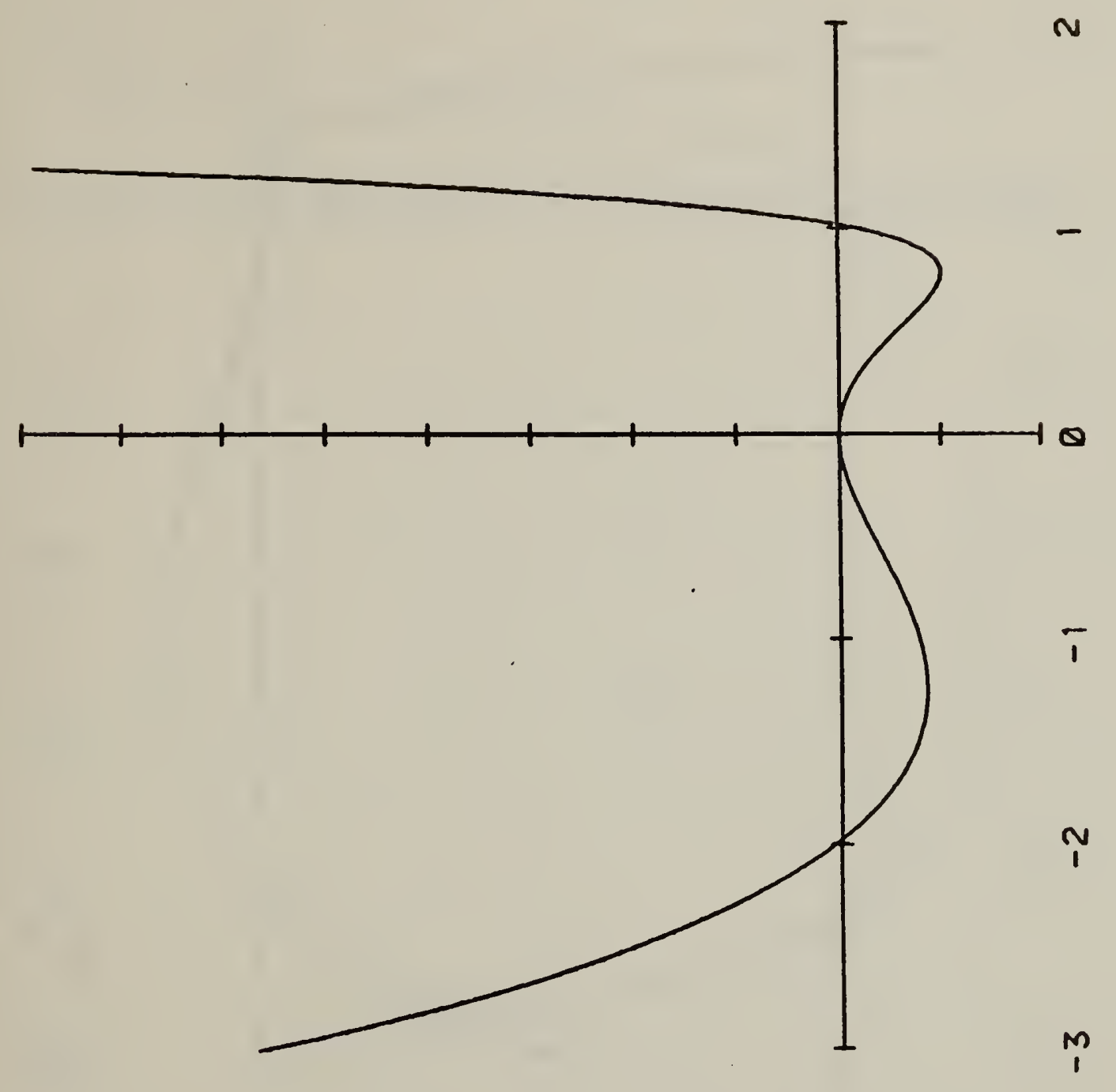




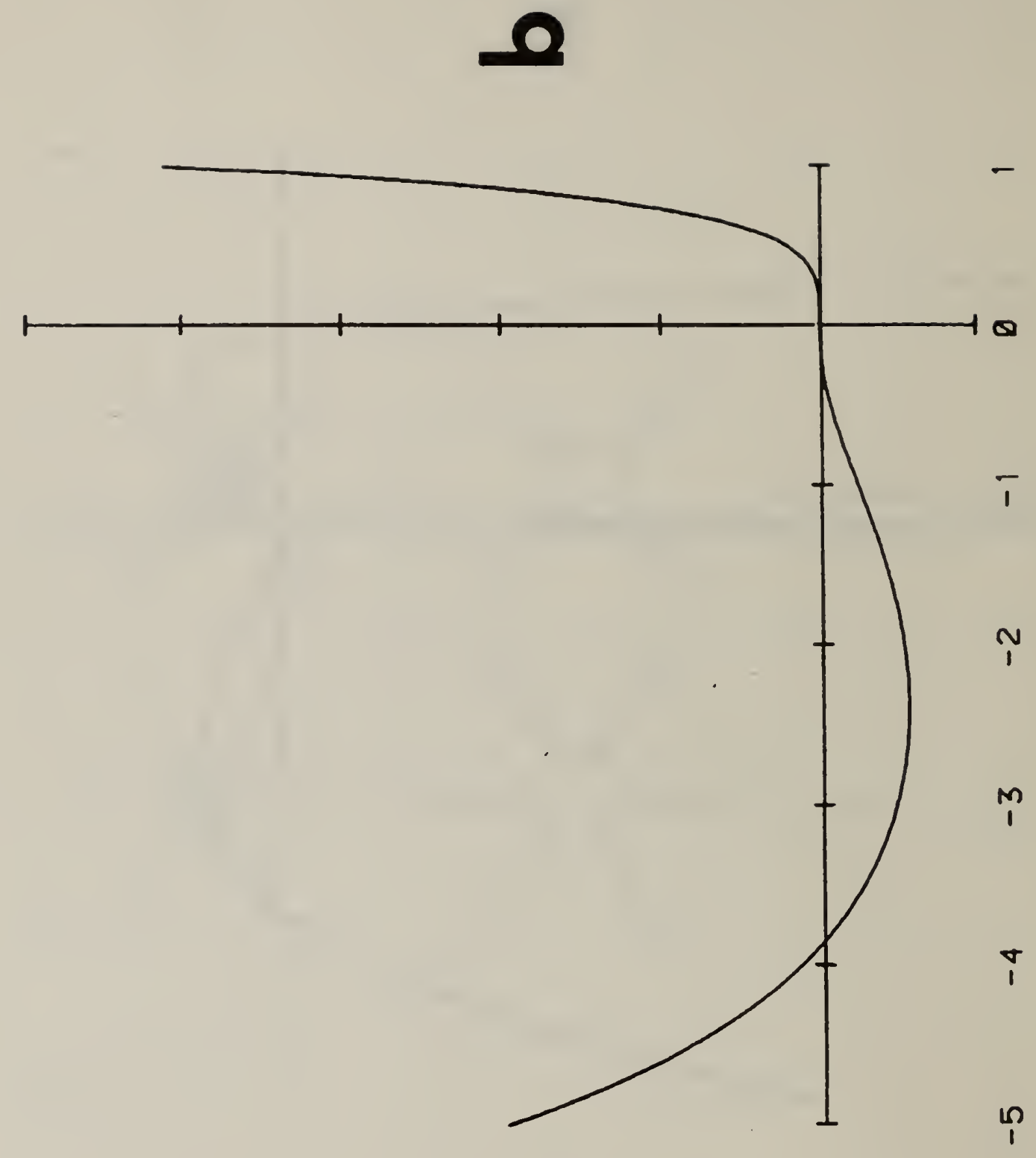




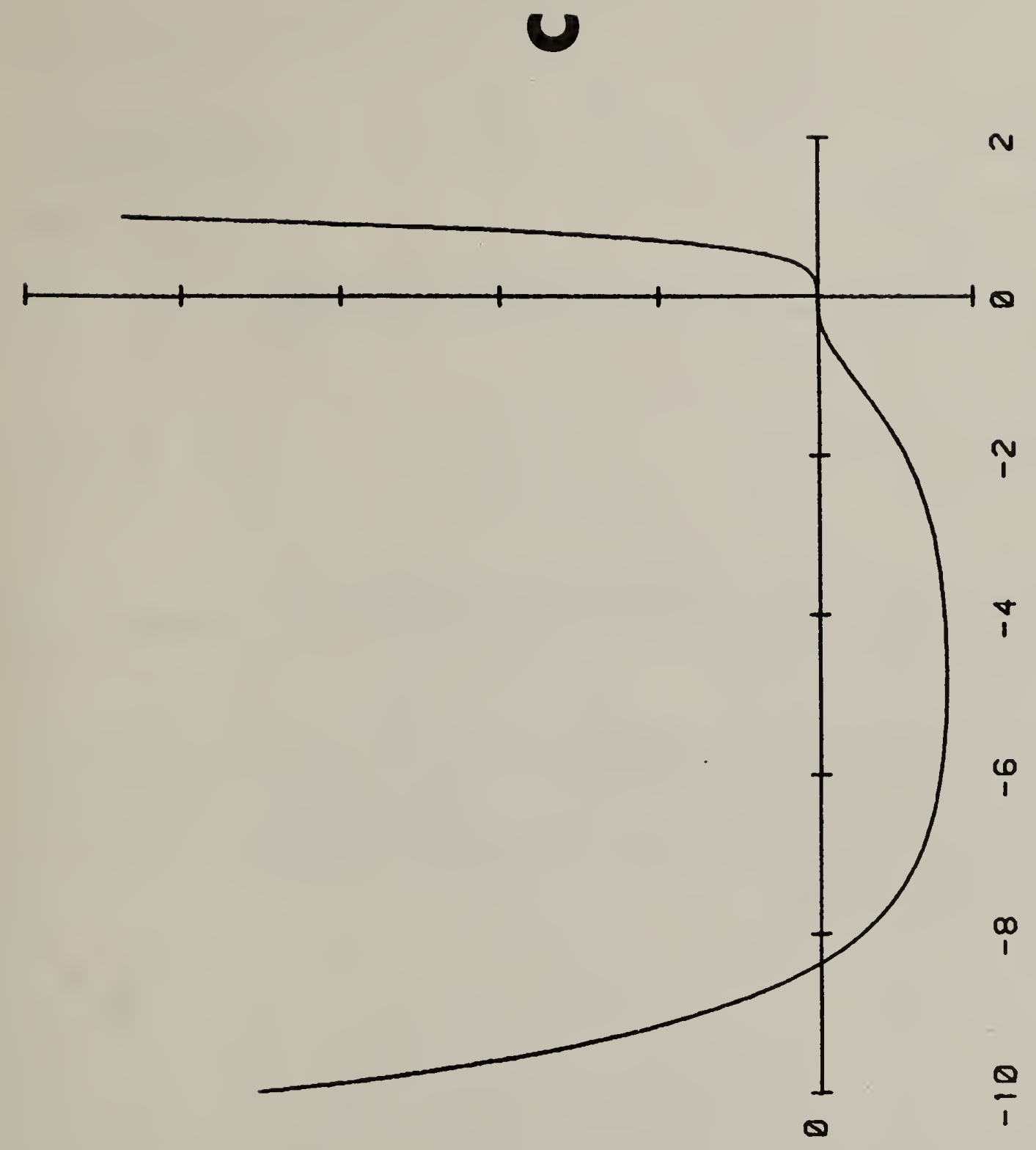


NBS.114A (REV. 2-8C)

U.S. DEPT. OF COMM.

BIBLIOGRAPHIC DATA

SHEET (See instructions)

1. PUBLICATION OR REPORT NO.

NBSIR $83-2759$

2. Performing Organ. Report No\& 3. Publication Date

August 1983

4. TITLE AND SUBTITLE

SIMPLE MODEL FOR THE QCD VACUUM

5. $A \cup T H O R(S)$

Michael Danos, Daniel Gogny and Daniel Irakane

6. PERFORMING ORGANIZATION (If joint or other than NBS, see instructlons)

7. ContracU Grant No.

NATIONAL BUREAU OF STANDARDS

DEPARTMENT OF COMMERCE

8. Type of Report \& Period Covered

WASHINGTON, D.C. 20234

9. SPONSCRING ORGANIZATION WAME AND COMPLETE ADORESS (Street, CHY, STOT,, ZIF)

10. SUPPLEMENTARY NOTES

[] Document describes a computer program; SF-185, FIPS Software Summary, is attached.

11. ABSTRACT (A 200-word or less factual summary of most significant information. If document includes a significant bibliogrophy or literature survey, mention it here)

By treating the high-momentum gluon and the quark sector as an in principle calculable effective Lagrangian we obtain a non-perturbative vacuum state for QCD as an infrared gluon condensate. This vacuum is removed from the perturbative vacuum by an energy gap. It is unstable below a minimum size and it has dia-electric and dia-magnetic character indicating expulsion of color-electric and -magnetic fields, i.e., it exhibits all the properties required for color confinement.

12. KEY WORDS (Six to twelve entries; alphobetical order: capitolize only proper names; and separate key words by semicolons)

bag constant; color confinement; confinement; gluon condensate; non-perturbative vacuum; QCD

13. AVAILABILITY

[X] Unlimited

For Official Distribution. Do Not Release to NTIS

Order From Superintendent of Documents, U.S. Government Printing Office, Washington, D.C. 20402.

X_ Order From National Technical Information Service (NTIS), Springfield, VA. 2216I
14. NO. OF

PRINTED PAGES

28

15. Price

$\$ 8.50$ 
in 
